\title{
Analysis of accuracy in optical motion capture - A protocol for laboratory setup evaluation
}

Patric Eichelberger ${ }^{1}$, Matteo Ferraro ${ }^{1}$, Ursina Minder ${ }^{1}$, Trevor Denton ${ }^{2}$, Angela Blasimann ${ }^{1}$, Fabian Krause $^{3}$, Heiner Baur ${ }^{1}$

${ }^{1}$ Bern University of Applied Sciences Health, Physiotherapy, Bern, Switzerland

${ }^{2}$ University of Michigan, School of Kinesiology, Ann Arbor, Michigan, USA

${ }^{3}$ University of Bern, Inselspital, Department of Orthopaedic Surgery, Bern, Switzerland

Keywords: optical motion capture, measurement error, accuracy, precision, uncertainty

\section{Submitting for Short Communication}

Wordcount: 2187 (Introduction through Discussion)

\section{Corresponding author}

Patric Eichelberger

Berner Fachhochschule

Murtenstrasse 10

3008 Bern

Switzerland

E-Mail address: patric.eichelberger@bfh.ch

Tel.: +41318484541 


\section{Abstract}

Validity and reliability as scientific quality criteria have to be considered when using optical motion capture $(\mathrm{OMC})$ for research purposes. Literature and standards recommend individual laboratory setup evaluation. However, system characteristics such as trueness, precision and uncertainty are often not addressed in scientific reports on 3D human movement analysis. One reason may be the lack of simple and practical methods for evaluating accuracy parameters of OMC.

A protocol was developed for investigating the accuracy of an OMC system (Vicon $\circledast$, volume $5.5 \times 1.2 \times 2.0 \mathrm{~m}^{3}$ ) with standard laboratory equipment and by means of trueness and uncertainty of marker distances. The study investigated the effects of number of cameras (6, 8 and 10), measurement height (foot, knee and hip) and movement condition (static and dynamic) on accuracy. Number of cameras, height and movement condition affected system accuracy significantly. For lower body assessment during level walking, the most favorable setting (10 cameras, foot region) revealed mean trueness and uncertainty to be $-0.08 \mathrm{~mm}$ and $0.33 \mathrm{~mm}$, respectively.

Dynamic accuracy cannot be predicted based on static error assessments. Dynamic procedures have to be used instead. The significant influence of the number of cameras and the measurement location suggests that instrumental errors should be evaluated in a laboratory- and task-specific manner. The use of standard laboratory equipment makes the proposed procedure widely applicable and it supports the setup process of OCM by simple functional error assessment. Careful system configuration and thorough measurement process control are needed to produce high-quality data. 


\section{Introduction}

Entertainment (Bregler, 2007), biomechanics (Sutherland, 2002), ergonomics (Wang et al., 2012) and sports (Bini et al., 2010) are possible fields of application of optical motion capture (OMC). Validity and reliability as scientific quality criteria have to be considered when using $\mathrm{OMC}$ in research. Miller and colleagues reported that the functional characteristics, namely accuracy, repeatability and resolution, had to be determined to report data with confidence (Miller et al., 2002). Accuracy, the systematic and random instrumental error, is often unspecified in scientific reports about 3D human movement analysis. One reason may be that manufacturers only provide rough accuracy specifications due to the fact that accuracy is influenced by many factors, such as camera setup, measurement and calibration volume, camera resolution, lighting conditions, etc. (Windolf et al., 2008). Windolf et al. reported that accuracy of OMC strongly depends on their individual setup and that accuracy and precision should be determined for an individual laboratory installation (Windolf et al., 2008). Published studies addressing the lack of accuracy information included examinations on system comparison (Ehara et al., 1997) (Richards, 1999), accuracy in angular (Vander Linden et al., 1992) and linear (Ehara et al., 1997) measurements and setup parameter influence (Windolf et al., 2008). Varying definitions of accuracy and precision limit the comparability between studies, but mostly accuracy was defined as mean absolute error and precision as mean standard deviation. This article follows the standards ISO 5725-1:1994 and JCGM 200:2012 which define the terms trueness, precision and uncertainty to quantify accuracy. Examination methods most often included special reference devices or positioning equipment, which is impractical for everyday use in the laboratory. Only Miller et al. focused on accuracy, repeatability and resolution to anatomical regions of interest (Miller et al., 2002). The standard ISO/IEC 17025:2005 state that test laboratories need methods to determine measurement uncertainty. As of today no simple and practical method exists to evaluate system accuracy with a close relation to anatomical regions of interest in biomechanical analysis. The purpose of this study was to develop a protocol for quantitative 3D OMC setup evaluation that is applicable within the process of setting up an OMC for measurement purposes.

\section{Materials and methods}

Accuracy was evaluated on different heights above the floor, according to anatomical regions of interest, namely ankle, knee and hip, in lower body gait analysis. Laboratory configurations under investigation consisted of six (C1-C6), eight (C1-C8) and ten (C1-C10) cameras (Vicon Bonita, 200 
$\mathrm{Hz}$, Vicon Motion Systems, Ltd., Oxford, UK) resulting in a measurement volume of $5.5 \times 1.2 \times 2.0 \mathrm{~m}^{3}$ (Figure 1). The fields of view from cameras $\mathrm{C} 7$ and $\mathrm{C} 8$ were fitted to the centrally placed force plate region (two AMTI OR6, AMTI Inc., Watertown, USA; sampling frequency $1 \mathrm{kHz}$ ) in order to enhance small magnitude foot measurement capabilities. Cameras $\mathrm{C} 9$ and $\mathrm{C} 10$ had a relatively wide-angle configuration with a field of view on the force plates and on the regions before and after the force plates. Camera calibration was made once with all ten cameras. Detailed information on the system parameters are provided as supplementary material. Repeated measurements from a static reference object and a dynamic reference object were taken in static and dynamic situations and at different heights with six (C1-C6), eight (C1-C8) and ten (C1-C10) cameras in operation. Static heights were chosen correspondingly to the heights of the anatomical regions of interest (Z0: floor - foot region, Z500: $500 \mathrm{~mm}$ above floor - knee region, Z1000: $1000 \mathrm{~mm}$ above floor - hip region) (Figure 3). Accuracy was estimated, analyzing measured marker distances of reference objects.

Insert Figure 1 about here

\subsection{Accuracy outcome measures}

Trueness expressed systematic deviations and was computed as mean marker distance error. Precision and uncertainty measured random deviations. Precision was the standard deviation of marker distances. Uncertainty measured the $95.5 \%$ confidence interval of the dispersion and equaled two times the precision.

$$
\begin{aligned}
& \text { Trueness: } \quad T=\frac{1}{N} \sum_{i=1}^{N}\left(l_{\text {ref }}-l_{i}\right)=\frac{1}{N} \sum_{i=1}^{N} e_{i} \\
& \text { Precision: } \quad P=\sqrt{\frac{1}{N-1} \sum_{i=1}^{N}\left(\bar{l}-l_{i}\right)^{2}} \\
& \text { Uncertainty: } \quad U=k \cdot P=2 \cdot P(95.5 \% \mathrm{Cl})
\end{aligned}
$$

\subsection{Data processing}

Data was analyzed with Matlab (Version R2013a) based on marker trajectories processed with Vicon® Nexus (Version 1.8.5). Processing in Vicon Nexus included marker trajectory processing operations commonly used in gait assessment: 3D reconstruction, trajectory smoothing, gap 
filling and exporting data to the c3d file format for further analysis (for details on software settings see supplementary material).

\subsection{Static procedure}

The Vicon® 5 Marker Wand, which is normally used for system calibration, served as the static reference object (marker diameter $14.62 \pm 0.04 \mathrm{~mm}$ ) (Figure 2). Markers and their coordinates $\mathbf{M}_{\mathbf{1}}$ $\left(S_{x}, 0,0\right), M_{2}\left(0,0, S_{z}\right)$ and $M_{3}\left(0, S_{y}, 0\right)$ were used to analyze the system accuracy in the $x-, y-$ and $z-$ direction. Reference marker center-to-center distances $\overline{M_{1} M_{2}}, \overline{M_{1} M_{3}}$ and $\overline{M_{2} M_{3}}$ were determined from five repeated caliper distance measurements, respectively. The reference $x-, y$ - and $z-$ distances $S_{x}(160.39 \pm 0.05 \mathrm{~mm}), S_{y}(118.73 \pm 0.05 \mathrm{~mm})$ and $S_{z}(110.17 \pm 0.05 \mathrm{~mm})$ were determined by solving a system of equations that related the measured marker distances with the marker coordinates. According to heights from the ankle-, knee- and hip joint, the static reference object was placed horizontally on the floor and $500 \mathrm{~mm}$ and $1000 \mathrm{~mm}$ above the floor (Figure 3). Origin and direction of the reference object's local $x$ - and $y$-axis were aligned with those from the global $x$ - and $y$-axis. Five 1-second intervals were captured for each height and respective accuracy outcome measures were calculated and averaged.

Insert Figure 2 about here

Insert Figure 3 about here

\subsection{Dynamic procedure}

The wrist tracking rod for full body assessment with Vicon's Plug-in Gait model (one marker with a diameter of $16.14 \pm 0.05 \mathrm{~mm}$ at each end) served as the reference object for the dynamic accuracy analysis (Figure 3). Reference marker center-to-center distance $D(95.53 \pm 0.05 \mathrm{~mm}$ ) was calculated from five repeated caliper measurements. The dynamic reference object was placed dorsally on the left foot (medial-lateral orientation), laterally on the left knee (anterior-posterior orientation) and on the sacrum (medial-lateral orientation) of the test person (Figure 3). The dynamic reference object was captured ten times while the subject was walking through the measurement volume in the longitudinal direction. To avoid gait direction bias, the subject passed the volume five times in the positive and five times in the negative x-direction. Accuracy outcome measures were calculated for the time interval between left initial contact and right toe off event and averaged over the ten repeated measurements. 


\subsection{Outcome analysis}

Outcomes were expressed for comparability reasons as relative bias and relative uncertainty.

Therefore, the accuracy outcome measures were normalized to respective reference marker

distances. Dynamic outcomes were compared with static outcomes, matching dynamic reference object orientations with the $x$-, $y$ - or z-direction from the static reference object at respective heights (Foot/Z0-Y, Knee/Z500-X, Hip/Z1000-Y).

\subsection{Statistical analysis}

Normal distribution of accuracy outcome measures was tested with the Kolmogorov-Smirnov test before statistical analysis. A 3-Way ANOVA with factors camera, measurement height and condition (static vs. dynamic) was performed to evaluate the influence of the number of cameras, measurement height and static or dynamic assessment on trueness and precision. Because the factors camera and measurement height both had three discrete levels, the Tukey-Kramer post-hoc test was applied to determine significantly different combinations. Statistical significance was set at $p<0.05$.

\section{Results}

The examined factors camera, measurement height and movement condition all influenced trueness and precision significantly $(p<0.001)$.

\subsection{Trueness}

Depending on the number of cameras, trueness for the static reference object was found to be between $2.00 \mathrm{~mm}(1.69 \%)$ and $2.30 \mathrm{~mm}(1.94 \%)$ for Z0-level, between $-0.11 \mathrm{~mm}(-0.07 \%)$ and -0.56 $\mathrm{mm}(-0.35 \%)$ for Z500-level and between $0.51 \mathrm{~mm}(0.43 \%)$ and $0.79 \mathrm{~mm}(0.66 \%)$ for Z1000-level. Trueness of dynamic reference object was found between: $-0.03 \mathrm{~mm}(-0.03 \%)$ and $-0.08 \mathrm{~mm}(-0.09 \%)$ for the foot-mounted position; $-0.38 \mathrm{~mm}(-0.40 \%)$ and $-0.90 \mathrm{~mm}(-0.94 \%)$ for the knee-mounted position; $-0.37 \mathrm{~mm}(-0.39 \%)$ and $0.16 \mathrm{~mm}(0.17 \%)$ for the hip-mounted position. The number of cameras and measurement height significantly influenced the trueness of static and dynamic measurements $(p<0.001)$. Overall trueness for static measurements was $0.84 \pm 1.05 \mathrm{~mm}$ (mean \pm SD) $(0.73 \pm 0.86 \%)$ and differed significantly from the overall trueness of dynamic measurement, which was $-0.23 \pm 0.35 \mathrm{~mm}(-0.24 \pm 0.36 \%)(p<0.001)$.

Insert Figure 4 about here 


\subsection{Uncertainty}

Static measurement uncertainty was found to be smaller than $0.07 \mathrm{~mm}(<0.06 \%)$ for all assessed configurations and was significantly influenced by the number of cameras and measurement height ( $p$ $<0.001)$. Dynamic measurement uncertainty laid between: $0.33 \mathrm{~mm}(0.34 \%)$ and $1.28 \mathrm{~mm}(1.34 \%)$ for the foot-mounted position; between $0.68 \mathrm{~mm}(0.72 \%)$ and $3.28 \mathrm{~mm}(3.47 \%)$ for the knee-mounted position; $0.81 \mathrm{~mm}(0.85 \%)$ and $1.25 \mathrm{~mm}(1.31 \%)$ for the hip-mounted position. The number of cameras and measurement height significantly influenced the uncertainty in dynamic measurements $(p<0.001)$. Overall uncertainty for dynamic measurements was $1.11 \pm 0.94 \mathrm{~mm}(1.16 \pm 0.99 \%)$ and significantly differed from overall uncertainty of static measurements $(p<0.001)$.

Insert Figure 5 about here

Insert Table 1 about here

\section{Discussion}

This study investigated the accuracy of an OMC system by means of trueness, precision and uncertainty of marker distances under the aspects of the number of cameras, measurement location and movement dynamics. Trueness of marker distances measured during lower-body stance phase assessments (level walking) was found to be in the range of $[-0.38,0.38] \mathrm{mm}$ with 10 cameras. Respective uncertainty was found to be $0.33 \mathrm{~mm}$ for the foot region, $0.74 \mathrm{~mm}$ for the knee region and $1.25 \mathrm{~mm}$ for the hip region. These rather small instrumental errors may not seem relevant for standard gait analysis with relatively large ranges of motion and dominating skin motion artifacts. However, they may become of special interest when analyzing small displacements such as, for example, intrinsic foot motion, simultaneously with full body kinematics. Commonly, a biomechanical assessment builds on models to calculate joint angles and it is left open to link the reported marker distance errors to such model outputs. Nevertheless, this study presented an instrumental error assessment method that is linked to anatomical regions of interest. Effects of measurement condition (static vs. dynamic) and number of cameras in operation were mutable in the sense of amount and direction of change of trueness and uncertainty. Changing from static to dynamic measurement condition improved the overall trueness but in contrast, increased the overall uncertainty. Changing the number of cameras did not significantly affect accuracy outcomes of static measurements. For the dynamic measurement condition, switching from 6 to 8 cameras in operation significantly improved the trueness on knee and 
hip as well as the uncertainty on foot and knee. Having 10 cameras in operation did not further improve any accuracy outcome measure. Furthermore, 8 cameras were sufficient to reach comparable accuracy. Except for the static uncertainty, which was very small in every case, inhomogeneous accuracy outcomes were observed for the different measurements heights which may be reasoned by changes in marker visibility conditions, such as shadowing or radial distortion effects towards field of view boundaries of a certain camera, or unequal measurement volume edge lengths. This is in contrast to previous findings, where consistent quasi-static accuracy parameters for the whole volume were reported (Miller et al., 2002). While the trueness did not show any relationship with measurement height, the uncertainty from the dynamic measurements with 8 and 10 cameras indicated an increased uncertainty with measurement height. The lowest uncertainty for the foot region confirms that we reached the goal of optimizing the uncertainty for measuring small displacements on the foot. When interpreting accuracy outcomes from varying heights, it has to be born in mind that also the measurement direction possibly has an influence, since orientation of the reference object was not the same on all heights. Nevertheless, the height dependency indicates measurement volume inhomogeneity and implies that accuracy should not be judged by single spot error assessments. Furthermore, it means that instrumental errors cannot be generally quantified based on knowledge of system parameters such as camera type, number of cameras used or size of measurement volume. Additionally, the specific task that should be captured may play a role: For example, system accuracy for measuring level walking without any obstacles occluding markers may be different than measuring a stair climbing task with potential marker visibility challenges. Our results indicate that instrumental errors should be evaluated in a laboratory- and taskspecific manner and underline the necessity of laboratory specific error assessments for having the whole measurement process under control and to guarantee high-quality research. The recommendation for setup specific instrumental error assessment is in consensus with previous findings (Miller et al., 2002) (Windolf et al., 2008) (Vander Linden et al., 1992) (Ehara et al., 1997). The significant accuracy outcome differences between static and dynamic measurements suggests, that the dynamic system accuracy cannot be predicted based on static error assessments and that dynamic procedures have to be used instead. The presented method fulfills the requirement for dynamic assessment and can be applied in direct relationship to anatomical regions of interest in biomechanical assessments. The use of standard laboratory equipment makes the procedure simple and widely applicable. It may support the setup process of OMC systems by task-specific and 
functional error assessment and is generally applicable to characterize arbitrary measurement volumes. Even though OMC is an established assessment tool for biomechanics, careful system configuration in combination with thorough measurement process control is needed to produce high quality results.

\section{Conflict of interest statement}

The authors state no conflicts of interest.

\section{Acknowledgements}

The investigation was carried out within the framework of the CurePPaC study, supported by the Swiss National Science Foundation (SNSF); Project funding Div. III Biology \& Medicine; Number 140928 (http://p3.snf.ch/project-140928).

\section{References}

Bini, R.R., Diefenthaeler, F., Mota, C.B., 2010. Fatigue effects on the coordinative pattern during cycling: Kinetics and kinematics evaluation. J Electromyogr Kines 20, 102-107.

Bregler, C., 2007. Motion capture technology for entertainment. IEEE Signal Processing Magazine 24, 160-158.

Ehara, Y., Fujimoto, H., Miyazaki, S., Mochimaru, M., Tanaka, S., Yamamoto, S., 1997. Comparison of the performance of 3D camera systems. Gait Posture 5, 251-255.

ISO, 5725:1994 Accuracy (trueness and precision) of measurement methods and results -- Part 1: General principles and definitions.

ISO/IEC, 2005. 17025:2005 General requirements for the competence of testing and calibration laboratories.

JCGM, 200:2012. International vocabulary of metrology - Basic and general concepts and associated terms (VIM).

Miller, C., Mulavara, A., Bloomberg, J., 2002. A quasi-static method for determining the characteristics of a motion capture camera system in a "split-volume" configuration. Gait Posture 16, 283-287.

Richards, J.G., 1999. The measurement of human motion: A comparison of commercially available systems. Human Movement Science 18, 589-602.

Sutherland, D.H., 2002. The evolution of clinical gait analysis - Part II - Kinematics. Gait Posture 16, 159-179.

Vander Linden, D.W., Carlson, S.J., Hubbard, R.L., 1992. Reproducibility and accuracy of angle

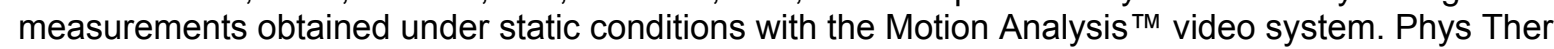
72, 300-305.

Wang, N., Kozak, K., Wan, J., Gomez-Levi, G., Strumolo, G., 2012. Enhancing Vehicle Ingress/Egress Ergonomics with Digital Human Models. Proceedings of the FISITA 2012 World Automotive Congress 195, 713-721.

Windolf, M., Gotzen, N., Morlock, M., 2008. Systematic accuracy and precision analysis of video motion capturing systems--exemplified on the Vicon-460 system. J Biomech 41, 2776-2780. 


\section{Tables}

Table 1: Test protocol with all tested combinations. With varying number of cameras, the dynamic reference object was captured at different anatomical regions and the static reference object at corresponding static heights (ZO: floor, Z500: $500 \mathrm{~mm}$ above floor, Z1000: $1000 \mathrm{~mm}$ above floor).

\section{Figures}

Figure 1: Motion capture setup (Vicon Motion Systems, Ldt., Oxford, UK) with eight cameras of type Bonita 3 and two cameras of type Bonita 10 (C7 \& C8). Cameras were mounted at a height of $2.6 \pm$ $0.2 \mathrm{~m}$ and provided a capture volume of $5.5 \times 1.2 \times 2.0 \mathrm{~m}^{3}$. Two centrally placed force plates (AMTI OR6, AMTI Inc., Watertown, USA; sampling frequency $1 \mathrm{kHz}$ ) provided initial contact (IC) and toe off (TO) event detection.

Figure 2: Static reference object with markers $M_{1}, M_{2}$ and $M_{3}$ that were used to calculate reference distances along $\mathrm{x}-\mathrm{y}$ - and $\mathrm{z}$-axis $\left(\mathrm{S}_{\mathrm{x}}, \mathrm{S}_{\mathrm{y}}, \mathrm{S}_{\mathrm{z}}\right)$.

Figure 3: Static \& dynamic reference objects at different heights.

Figure 4: Relative bias with SD for all measured conditions.

Figure 5: Relative uncertainty with SD for all measured conditions. 
Figure1

C3
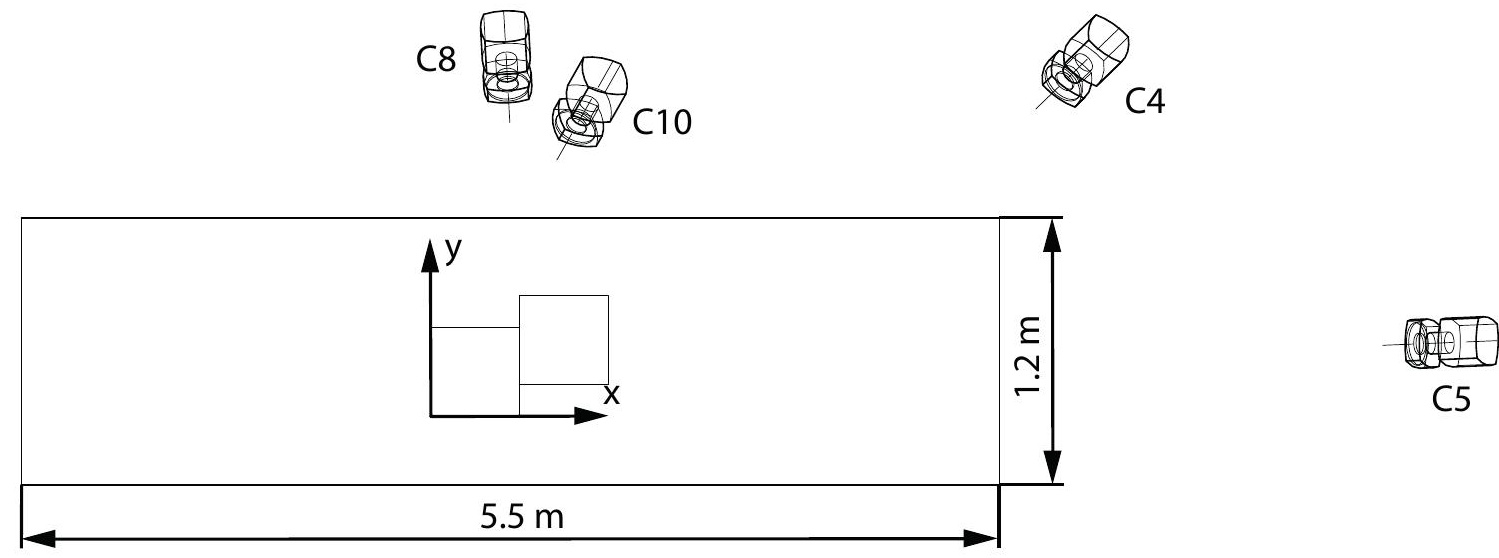

C5

पि口

$\mathrm{C} 2$

$5.5 \mathrm{~m}$

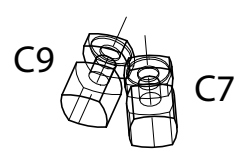




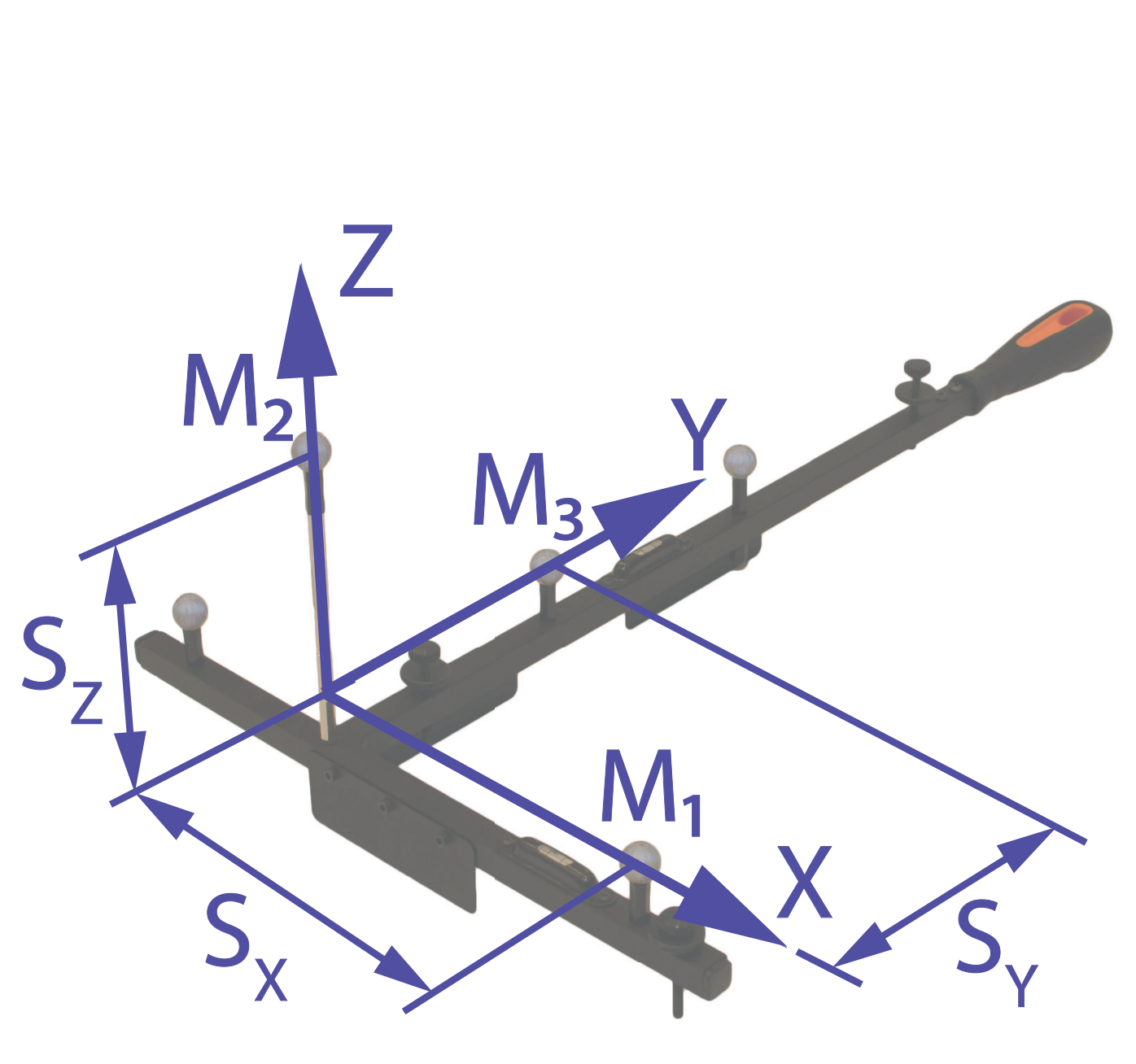

Figure2

Figure2

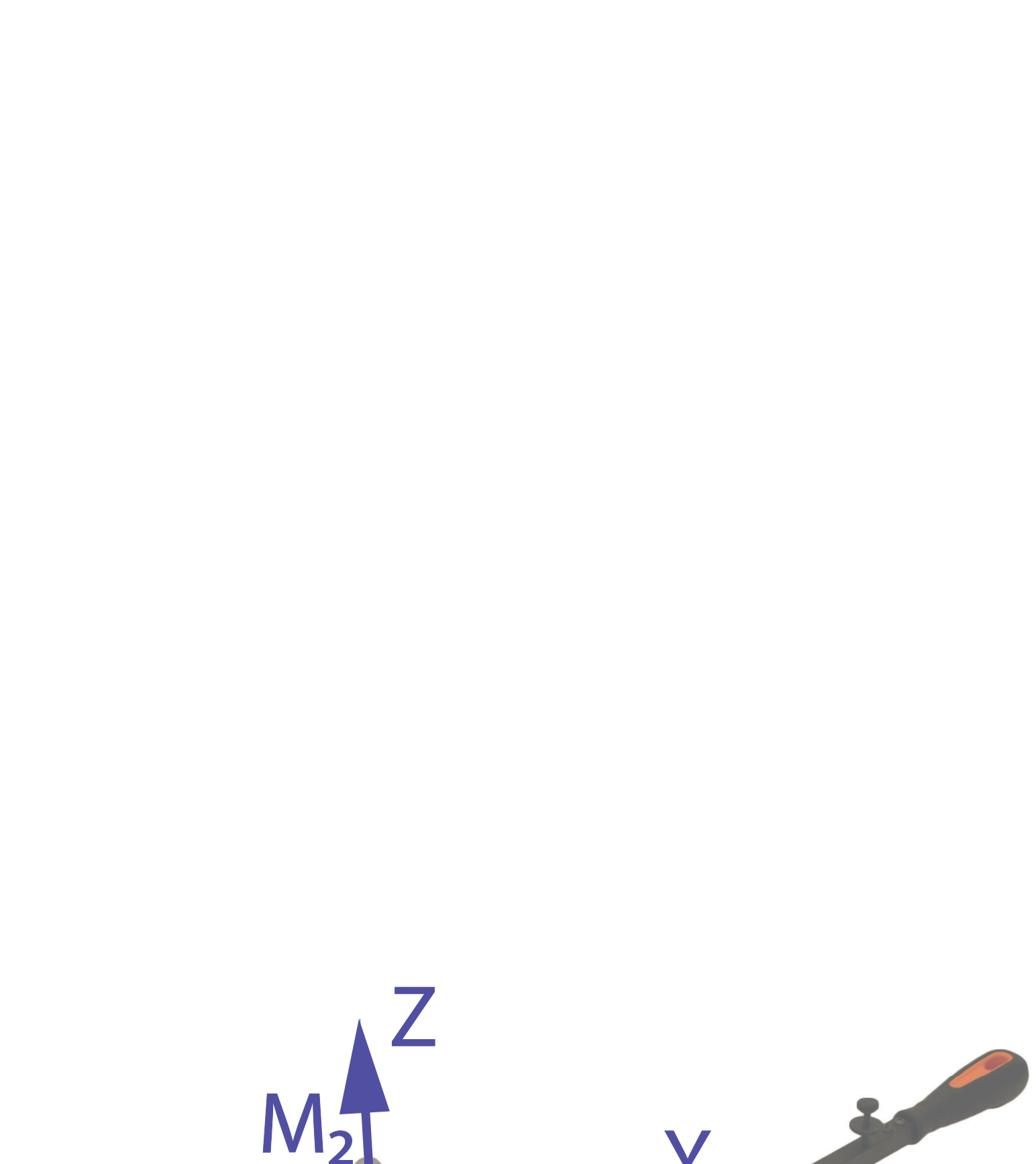

Figure2

(1)
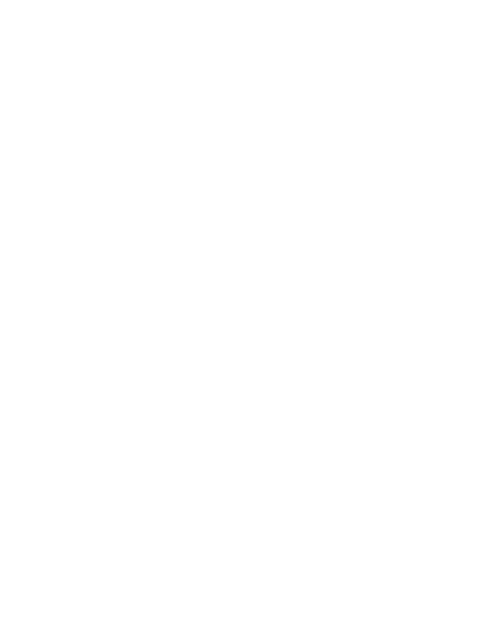
Dynamic

ฮ)

드
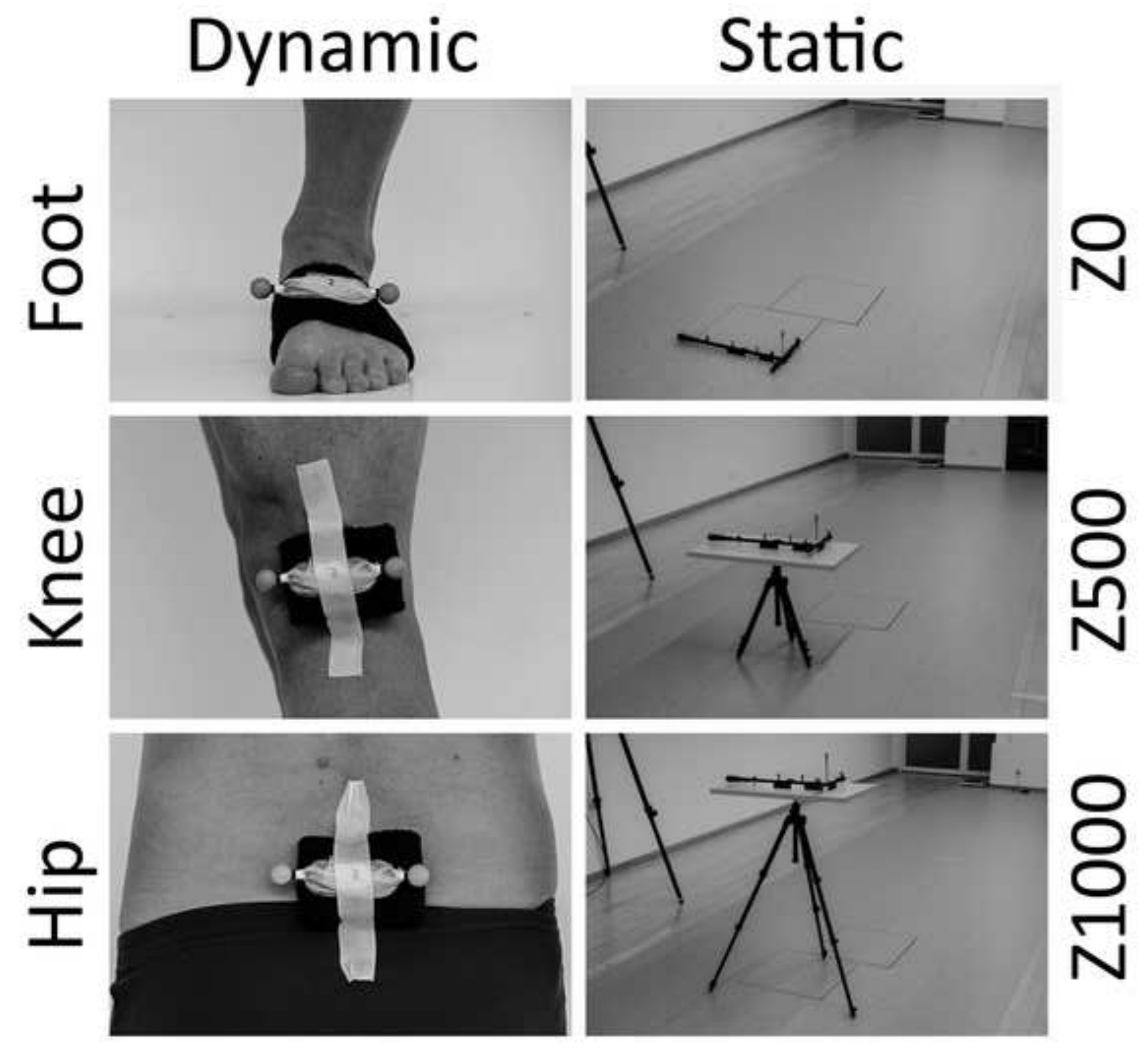

Static

$N$

ڤ


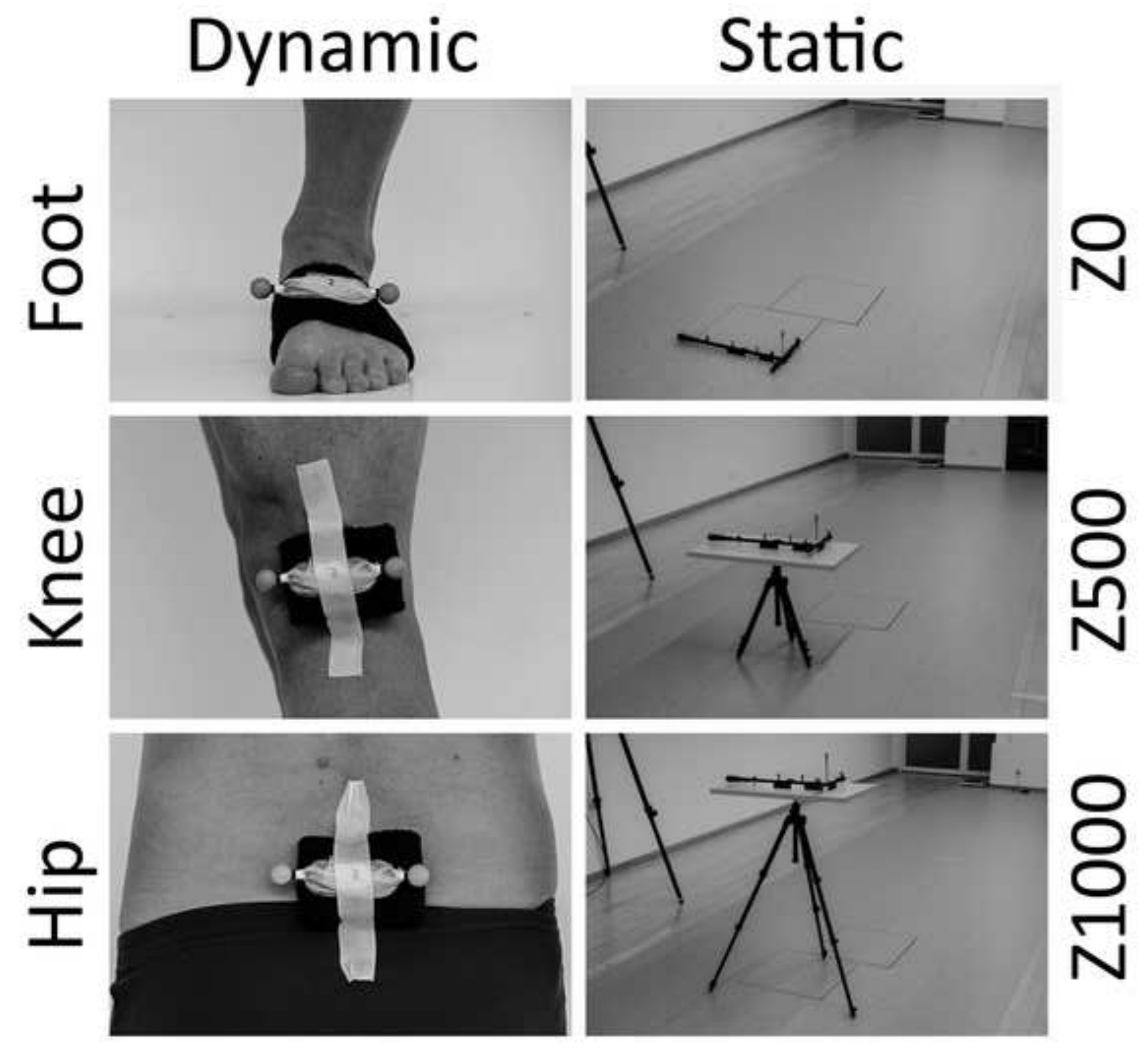


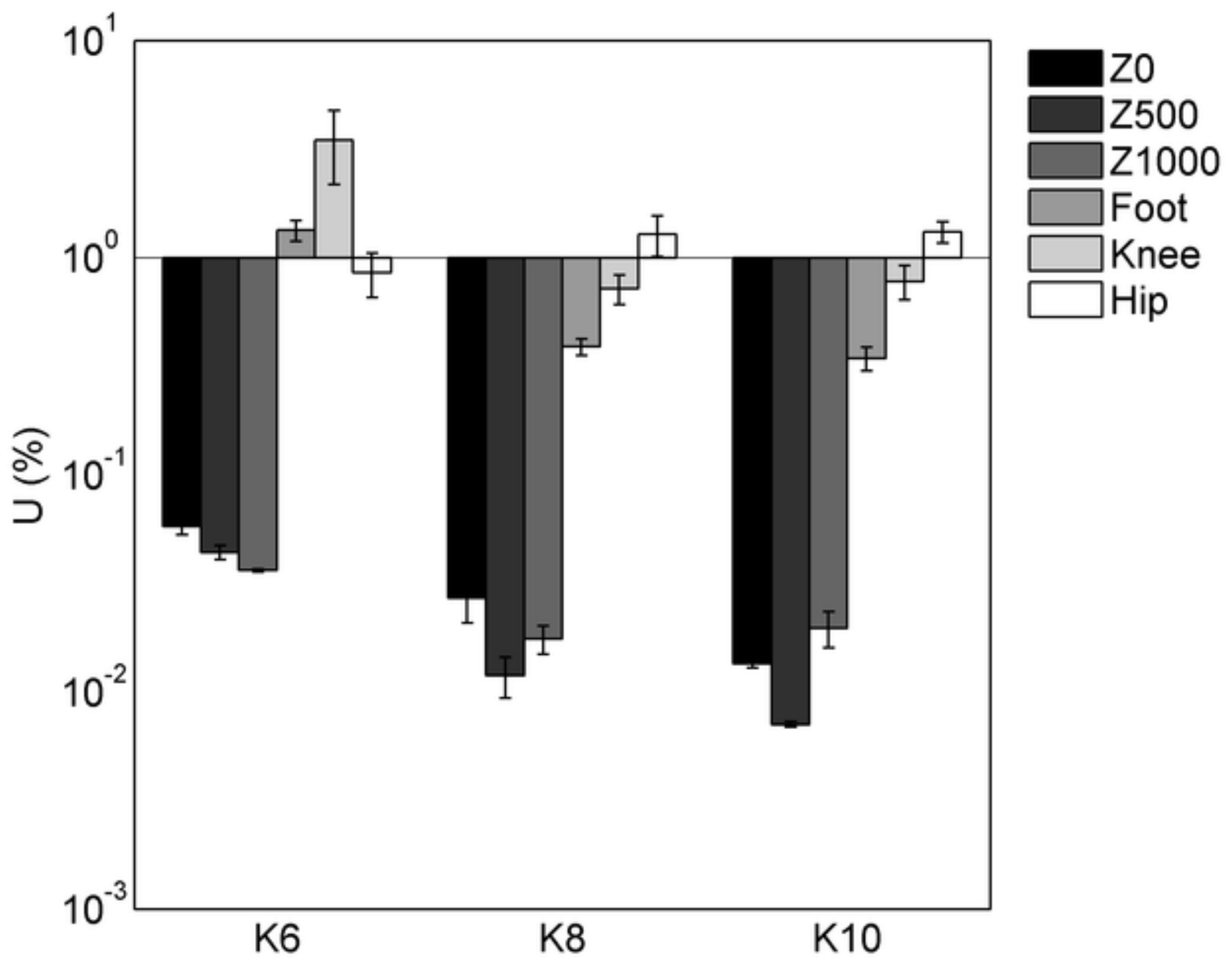




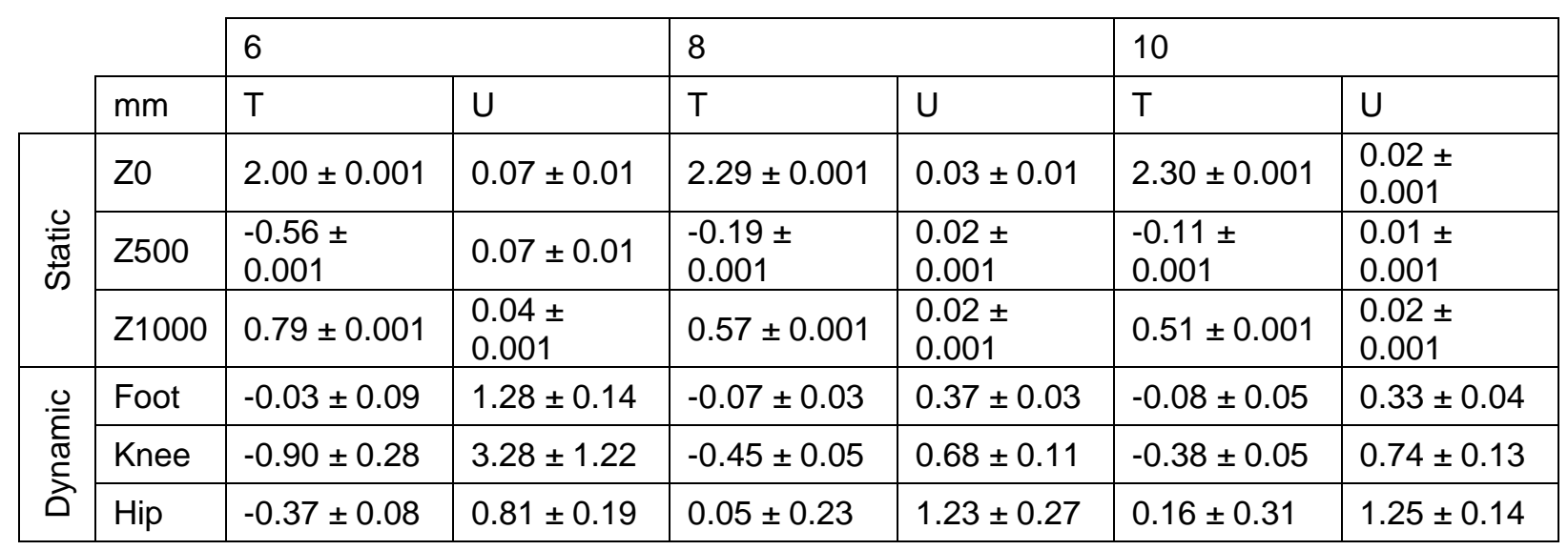

